\title{
Deep optical observations of the fields of two nearby millisecond pulsars with the VLT`
}

\author{
F. K. Sutaria ${ }^{1}$, A. Ray ${ }^{2}$, A. Reisenegger ${ }^{3}$, G. Hertling ${ }^{3}$, H. Quintana $^{3}$, and D. Minniti ${ }^{3}$ \\ ${ }^{1}$ Dept. of Physics and Astronomy, The Open University, Milton Keynes, UK \\ 2 Tata Institute of Fundamental Research, Mumbai, India \\ 3 Dept. of Astronomy and Astrophysics, Pontificia Universidad Católica de Chile, Santiago, Chile
}

Received 3 January 2003 / Accepted 7 May 2003

\begin{abstract}
We report on deep VLT observations of the fields of two nearby, isolated millisecond pulsars, PSR J1744-1134 and PSR J1024-0719. Both objects are old neutron stars with characteristic age $\tau \geq 10^{9} \mathrm{yr}$ and have relatively high spin-down flux. They have been detected earlier as X-ray sources by ROSAT HRI observations and were considered good candidates for non-thermal emission in the optical bands. Our observations set an upper limit of $B=26.9, V=26.3$ and $R=26.0$ for PSR J1744-1134. In the case of PSR J1024-0719, we find two faint objects near the radio position of the pulsar. Using multiband photometry from the VLT and spectroscopy carried out with the Magellan I telescope, we discuss the nature of the brighter object and the possibility of the fainter one being the optical counterpart of PSR J1024-0719. We consider the implications of our findings for both pulsars in the context of theoretical models of high-energy emission from old pulsars.
\end{abstract}

Key words. stars: neutron - pulsars: general - pulsars: individual: PSR J1024-0719, PSR J1744-1134 radiation mechanisms: non-thermal - radiation mechanisms: thermal

\section{Introduction}

Optical pulses from the Crab pulsar were detected by Cocke et al. (1969) within a year of the discovery of the pulsar in the radio band (Staelin \& Reifenstein 1968) and the inauguration of the field of pulsars by S. Jocelyn Bell and collaborators (Hewish et al. 1968). Since then, the number of pulsars discovered in the radio band has gone up to nearly 1400 (ATNF pulsar catalog), but the number of pulsed counterparts detected in the higher energy bands remains small. To date, the number of radio pulsars with detected optical pulses remains at 5 (Shearer \& Golden 2002), and with X-ray pulses 22, the latter including 6 millisecond pulsars (Becker \& Aschenbach 2002).

The pulsed high-energy radiation from an ordinary rotation-powered (isolated) pulsar is a combination of differing amounts of three spectral components: 1) power-law emission resulting from particles accelerated in the magnetosphere, 2) a soft black-body component from the surface of a cooling neutron star, and 3) a hard thermal component associated with heated polar caps bombarded by energetic particles. Measurements of the radiative fluxes and spectra in different bands can constrain the relative weights of these components,

Send offprint requests to: A. Reisenegger,

e-mail: areisene@astro.puc.cl

* Based on observations with the ESO 8.2m VLT-Antu (UT1) Telescope under Programs 66.D-0448 and 67.D-0538. providing observational input to the nature of the underlying mechanism of pulsar radiation (see e.g. Pavlov et al. 1996).

Millisecond pulsars (MSPs) are a special class, not only because of their short spin periods, but also because of their smaller surface magnetic field, old age, and evolutionary history of spin-up due to accretion of mass and angular momentum from a binary companion (Alpar et al. 1982). In the process, the star may have been resurrected from a state of hibernation into one of detectable pulsed radio emission. They represent a considerable extension of the parameter space of classical pulsars, so different radiation mechanisms may well be active in their magnetospheres. The old age of the MSPs, on the other hand, implies that they have almost certainly radiated away any "fossil" heat from their original collapse or from their accretion epoch. Thus, any thermal emission must be due to external reheating from the magnetosphere or to internal dissipation mechanisms, most likely related to the star's slowing rotation rate (see, e.g., Cheng et al. 1992; Reisenegger 1995; Larson \& Link 1999, and references therein).

While MSPs with binary companions are interesting in their own right from the evolutionary angle, radiation from the companions, even if they are low-mass old white dwarfs, "contaminates" the faint optical radiation intrinsic to the pulsar itself. It is therefore of interest to concentrate on the isolated pulsars to study their radiative properties in these bands. Isolated MSPs are relatively rare objects - till mid-2002, only 10 out of $\sim 57$ known Galactic MSPs (outside of globular 
clusters) are isolated. Due partly to the lack of deep observations, no MSPs have been detected so far in the optical. The advent of the VLT class of telescopes is therefore a crucial technological development for these faint objects.

In this paper, we present the results of deep VLT observations of two southern, isolated MSPs, PSR J1024-0719 and PSR J1744-1134, which are among the closest MSPs discovered so far and which have also been detected in the X-ray band by ROSAT. Identification of candidate objects in the optical band is the first step towards searches for optical pulsations from a pulsar. These pulsars have some of the highest spin-down fluxes $\left(\dot{E}_{\text {rot }} / 4 \pi d^{2}=I \Omega \dot{\Omega} / 4 \pi d^{2}\right)$ among MSPs, an indicator of their propensity to produce high-energy radiation. Because of their proximity, as well as the low extinction in their direction, they are among the best candidates for optical detection. Two other nearby MSPs with low extinction in their directions, PSR J0030+0451 (Koptsevich et al. 2003) and PSR J2124-3358 (Mignani \& Becker 2003) have also been observed with the VLT, but no optical counterparts of the radio pulsars have been found. In Sect. 2, we discuss what is known about these two pulsars in the X-ray and radio bands. We next present, in Sect. 3, the VLT service mode observations and their data analysis including accuracy considerations for astrometry. In Sect. 4, we discuss the results obtained from the VLT data. We discuss the nature of the optical objects near PSR J1024-0719 in Sect. 5 and supplement our discussion of a star near PSR J1024-0719 with a spectroscopic observation with the Magellan I Baade $6.5 \mathrm{~m}$ telescope at Las Campanas Observatory. We compare our results in the context of theoretical models of pulsar radiation in Sect. 6. In Sect. 7, we discuss the multi-band optical results together with the highenergy and radio emission from the pulsars and compare these with those from slower pulsars. Finally, our conclusions are given in Sect. 8.

\section{Radio and X-ray properties of the two pulsars}

Both PSR J1744-1134 and PSR J1024-0719 were first detected in the radio bands as isolated MSP with periods $P=4.07$ and $5.16 \mathrm{~ms}$, respectively (Bailes et al. 1997). In Table 1 we quote some characteristics of these objects relevant to our observations and their interpretation. The astrometric data and the proper motions were determined by radio timing observations (Toscano et al. 1999a).

The radio pulse profile of PSR J1744-1134 is narrow and sharply peaked, with a duty cycle of $\sim 10 \%$, while PSR J1024-0719 exhibits a broad, multiple-peaked profile with a duty cycle of $\sim 50 \%$. The radio and X-ray emission are most likely based on different physical mechanisms: curvature radiation from $e^{ \pm}$pairs near the NS surface (Ruderman \& Sutherland 1975), and synchrotron radiation from energetic particles in the outer gap (Cheng et al. 1986), respectively. However, at least in the case of PSR B1821-24 (another MSP), RXTE observations (Rots et al. 1997) show that the leading edge of the most energetic pulse coincides with the radio pulse profile at $800 \mathrm{MHz}$, possibly implying a common site for the origin of the two pulses.
Table 1. Characteristics of PSRs J1024-0719 and J1744-1134 (BT99, Toscano et al. 1999a; Toscano et al. 1999b).

\begin{tabular}{ccc}
\hline \hline Property & PSR J1024-0719 & PSR J1744-1134 \\
\hline Period $(P)[\mathrm{ms}]$ & 5.16 & 4.07 \\
$\dot{P}\left[10^{-20} \mathrm{~s} \mathrm{~s}^{-1}\right]$ & $1.873(5)$ & $0.89405(9)$ \\
$\dot{E}_{\mathrm{rot}}\left[\mathrm{erg} \mathrm{s}^{-1}\right]$ & $5.25 \times 10^{33}$ & $1.90 \times 10^{33}$ \\
$d[\mathrm{kpc}]$ & 0.200 & 0.357 \\
$\tau\left[10^{9} \mathrm{yr}\right]$ & 27 & 9.1 \\
$B\left[10^{8} \mathrm{G}\right]$ & 1.3 & 1.7 \\
RA $(\mathrm{J} 2000)$ & $10^{\mathrm{h}} 24^{\mathrm{m}} 38^{\mathrm{s}} .7040(1)$ & $17^{\mathrm{h}} 44^{\mathrm{m}} 29^{\mathrm{s}} .390963(5)$ \\
Dec $(\mathrm{J} 2000)$ & $-07^{\circ} 19^{\prime} 18^{\prime \prime} .849(3)$ & $-11^{\circ} 34^{\prime} 54^{\prime \prime} .5746(5)$ \\
P. M. $(\mathrm{RA})$ & $-41(2)$ & $18.72(6)$ \\
{$\left[\mathrm{mas} \mathrm{yr}{ }^{-1}\right]$} & & \\
P. M. $(\mathrm{Dec})$ & $-70(3)$ & $-9.5(4)$ \\
{$\left[\mathrm{mas} \mathrm{yr}{ }^{-1}\right]$} & & 50434.0 \\
Epoch $(\mathrm{MJD})$ & 50456.0 & \\
$L_{\mathrm{X}}(0.2-2.4 \mathrm{keV})$ & $1 \times 10^{29}(d / 200 \mathrm{pc})^{2}$ & $4 \times 10^{29}(d / 360 \mathrm{pc})^{2}$ \\
{$\left[\mathrm{erg} \mathrm{s}{ }^{-1}\right]$} & & \\
\hline
\end{tabular}

The two pulsars are similar in that both their spin-down ages are $\tau \equiv P /(2 \dot{P})>10^{9}$ yr (Table 1). Thus, both are so old that non-thermal magnetospheric emission can be expected to dominate over any thermal effects, unless strong re-heating occurs. Some uncertainty exists over the distance estimate to the two pulsars. An upper limit to the distance of PSR J1744-1134, based on the assumption that the period derivative is entirely due to the transverse motion of the pulsar ("Shklovskii effect"; Shklovskii 1970), is 1910 pc (Toscano et al. 1999a). Based on parallax measurements of PSR J1744-1134, Toscano et al. 1999b estimate the distance $d$ as $357_{-35}^{+43}$ pc. This number decreases to $166 \mathrm{pc}$ (Bailes et al. 1997) if $d$ is estimated using the measured value of the dispersion measure (DM) and values of electron densities from the Taylor \& Cordes (1993) model. However, it is to be noted that, while this model adequately describes the properties of interstellar medium (ISM) at $d>1 \mathrm{kpc}$, it does not account properly for fluctuations in the local ISM. For our purposes, we use a distance estimate of $0.357 \mathrm{kpc}$ for PSR J1744-1134. In the case of PSR J1024-0719, an upper limit $d<0.226 \mathrm{kpc}$ is obtained from the Shklovskii effect, while $d=0.35 \mathrm{kpc}$ is obtained by using DM and the Taylor and Cordes model (Toscano et al. 1999a). We shall adopt a value $d=0.2 \mathrm{kpc}$ for PSR J1024-0719 in this paper, unless specified otherwise.

X-ray counterparts for both pulsars were discovered by the ROSAT HRI (Becker \& Trümper 1999; hereafter BT99). Because both pulsars are quite faint in this band, no timing observations could be done, and the counterparts were identified by their proximity to the radio positions. Assuming a photon spectral index $\alpha=2$, these authors find that the unabsorbed X-ray luminosity of PSR J1744-1134 (scaled to our adopted distance) is $L_{\mathrm{X}}=4 \times 10^{29}(d / 0.36 \mathrm{kpc})^{2} \mathrm{erg} \mathrm{s}^{-1}$, and that of PSR J1024-0719 is $L_{\mathrm{X}}=1 \times 10^{29}(d / 0.2 \mathrm{kpc})^{2} \mathrm{erg} \mathrm{s}^{-1}$. The X-ray to spin-down luminosity ratios $L_{\mathrm{X}} / \dot{E}_{\text {rot }}$ are then $2 \times 10^{-4}$ and $5 \times 10^{-5}$, respectively, somewhat below the general 
Table 2. Observation summary for VLT FORS1 CCD photometry.

\begin{tabular}{ccccc}
\hline \hline PSR & Filter & $\begin{array}{c}\text { Exposure } \\
\text { time } \\
{[\mathrm{s}]}\end{array}$ & $\begin{array}{c}\text { Mean } \\
\text { air } \\
\text { mass }\end{array}$ & $\begin{array}{c}\text { Mean } \\
\text { Seeing } \\
{\left[{ }^{\prime \prime}\right]}\end{array}$ \\
\hline $\mathrm{J} 1744-1134$ & $B$ & $\begin{array}{c}20 \times 600 \\
2 \times 540\end{array}$ & 1.04 & 0.725 \\
& $V$ & $\begin{array}{c}2 \times 06 \\
+3 \times 600\end{array}$ & 1.095 \\
& & & \\
& $R$ & $2 \times 540$ & 1.08 & 0.795 \\
& & $+3 \times 600$ & & \\
\hline $\mathrm{J} 1024-0719$ & $U$ & $1 \times 900$ & 1.2 & 0.63 \\
& $V$ & $3 \times 120$ & 1.3 & 0.97 \\
& $R$ & $3 \times 180$ & 1.2 & 0.74 \\
& $I$ & $3 \times 120$ & 1.2 & 0.71 \\
\hline
\end{tabular}

relation that Becker \& Trümper (1997) found statistically among high-energy pulsars, $L_{\mathrm{X}} / \dot{E}_{\mathrm{rot}} \sim 10^{-3}$.

\section{The VLT imaging observations}

The first step towards identifying an optical counterpart involves locating an optical object in near positional coincidence with the radio (timing) position of the MSP. The field centered on PSR J1744-1134 was observed by the ESO 8.2m Very Large Telescope Antu (VLT-UT1) with the FORS1 CCD in the narrow-field imaging mode in the Bessel $B, V$, and $R$ bands on four nights during April 2001 (MJD 52017-52027). The field of PSR J1024-0719 was observed by the same instruments/mode in the $U, V, R$, and $I$ bands within less than one hour in March 2001 (MJD 51996). Both sets of observations were carried out in the service mode. Although we had requested comparable exposures for several MSPs with the VLT, the final exposures attained in the service mode within the seeing and other requirements in the respective observing cycles differed substantially for the two targets described here. A brief summary of the observations is provided in Table 2.

\subsection{The observation}

Our initial examination of the Digital Sky Survey (DSS) plates in the vicinity of PSR J1744-1134 showed no bright objects. However, the deep VLT images show a star with $V=21.3$ about 2.'0 from the position of the pulsar. Thus, in order to strike a balance between deep exposure and good seeing, of the twenty-three ten-minute exposures with Bessel $B$, we rejected the three exposures with the worst seeing taken on 2001 April 21, thus reducing the available photometry time to $3.33 \mathrm{hr}$. The available exposure time in each of the $V$ and $R$ filters was $48 \mathrm{~min}$. The median seeing in the $B, V$, and $R$ filters was $0.7,1$.' 1 , and $0 .{ }^{\prime} 8$, respectively.

For PSR J1024-0719, the available exposure time was $13.33 \mathrm{~min}$ in $U, 6 \mathrm{~min}$ in both $V$ and $I$, and $9 \mathrm{~min}$ in $R$ (see Table 2). The median seeing in the $U, V, R$, and $I$ frames was $0.6,11^{\prime \prime} 0,0 . .7$, and 0.77 respectively.

\subsection{Data reduction}

The photometric analysis for both objects was done using the MIDAS (automated) pipeline processed data. The pipeline processing ensures that each frame is checked for over-exposure and suitably bias-subtracted and flat-fielded, using the master bias and flats generated from calibration observations made on each night. Finally, the prescan and over-scan regions are removed from the science images used in this analysis. The image processing was done using the IRAF ${ }^{1}$ software. With the exception of the single image in the $U$-band, the reduced images in each filter were aligned and median-combined using a cosmic-ray rejection algorithm. For both PSR J1024-0719 and PSR J1744-1134, a $V \sim 20-21$ star is close to the pulsar position, so the photometric analysis of the combined images was carried out using PSF-subtraction photometry (Massey \& Davis 1992). In order to ensure that the wings of the PSF (point spread function) of nearby stars are subtracted out cleanly, we constructed a PSF for each frame, using only the brightest unsaturated stars, which were of magnitude a little brighter or close to that of the "contaminating star". In general, we find that the PSF of the FORS1-CCD is positiondependent and is best fit by the IRAF moffat 25 or penny 1 functions with 2 nd order variability in $X$ and $Y$. Aperture correction was done on the CCD magnitudes, and the final magnitudes of all objects observed in the vicinity of the pulsar's radio position were determined by comparison with standard stars. The standard stars used were from Landolt (1992) field SA109 for PSR J1024-0719, and fields SA109, SA110, and MARKA for PSR J1744-1134, observed on the same nights.

\subsection{Astrometry}

Correcting for the proper motion, the radio timing position of PSR J1744-1134 at the epoch of the VLT observations was RA $=17^{\mathrm{h}} 44^{\mathrm{m}} 29.39638(3)$, Dec $=-11^{\circ} 34^{\prime} 54^{\prime \prime} .616(2)$, and that of PSR J1024-0719 was RA $=10^{\mathrm{h}} 24^{\mathrm{m}} 38^{\mathrm{s}} .6925(6)$, Dec $=-07^{\circ} 19^{\prime} 19^{\prime} .14(1)$.

Astrometric corrections to the observed CCD positions were carried out by comparison with both the USNO-A 2 cat$\operatorname{alog}^{2}$ of astrometric standards (Monet et al. 1998) and the HST Guide Star Catalog II (GSCII version 2.2) ${ }^{3}$. We report the results based on the more recent GSCII catalog, where the epoch of observation was from 1983-1986. Since the observations were carried out in the high-resolution mode for better sampling the PSF, the field of view (FOV) was halved and the number of GSCII astrometric standards in the FOV of PSR J1024-0719 was only 6. The crowded field of PSR J17441134 provided 14 astrometric standards within 1.'5 of the pulsar position. The FORS1 CCD pixel coordinates of the (GSCII) reference stars were obtained from our photometric analysis,

\footnotetext{
${ }^{1}$ IRAF is distributed by the National Optical Astronomy Observatories, which is operated by the Association of Universities for Research in Astronomy, Inc. (AURA) under cooperative agreement with the National Science Foundation.

${ }^{2}$ http://www.nofs.navy.mil/projects/pmm/catalogs. html

${ }^{3}$ http://www-gsss.stsci.edu/support/data_access.htm
} 
and they were converted to (measured) RA and Dec using the ASTROM package supplied with the STARLINK ${ }^{4}$ software.

For the field of PSR J1024-0719, the combined rms error from PSF-fitting and transformations was 0 .' 12 in RA and 0 .' $^{\prime} 16$ in Dec. The average error in the positions of the relevant stars in the GSCII catalog is 0.39 in both directions (available from the catalog web-tool address given below. See also Deutsch (1999) for the empirical uncertainty estimators for the reference catalogs with respect to the ICRF.). Thus, incorporating the error in the measurement of the proper motion, we estimate the total astrometric error in the observation of this field at $0 . " 42$ in RA and 0.' 43 in Dec. For PSR J1744-1134, the average error in the positions of the GSCII catalog stars in the field is 0.'28 in RA and 0.'30 in Dec, and the error in the PSF fitting and transformations is $0 . \prime 16$ in RA and 0.'14 in Dec. In this case, the total astrometric error in the position is 0.'34 and 0.'33 in RA and Dec, respectively. We note that astrometry relative to the USNO-A2 catalog provides similar results, though the errors are slightly larger.

Since we use the radio pulsar positions to search for their optical counterparts, we need to consider the accuracy of the radio timing position with respect to the position in the optical frame. The GSCII catalog was calibrated using the Hipparcos and Tycho frames of reference, which are tied to the International Celestial Reference Frame (ICRF, tied to the radio Very Long Baseline Interferometry, Ma et al. 1998).

An estimate of the absolute accuracy of pulsar radio timing positions was made by Fomalont et al. (1992) (and Fomalont et al. 1997) through comparison with positions determined by interferometry. In general, they found the RA and Dec position offsets derived from timing and interferometric positions to be a function of the position of the target in the sky. Fomalont et al. (1992) state that, while there are no significant offsets between the coordinate frames in a global comparison of VLA positions and timing positions, there is a mean scatter of about $0{ }^{\prime} 8$ in each coordinate (RA and Dec).

If we add this to the above astrometric errors, then for both pulsars the total astrometric error in both RA and Dec would be $\approx 0.9$, giving an error circle of radius $\approx 1 .{ }^{\prime \prime} 3$. Therefore, any optical star-like emission within about 1 .' 5 of the radio timing position should be taken with interest for further scrutiny, especially for time resolved photometry.

\section{Results of imaging observations}

The broadband magnitudes in multiple optical bands and astrometric positions of objects within 2 .'0 of the radio timing positions of our two target MSPs are reported in Table 3. We do not detect any object within 1.'5 of PSR J1744-1134. An image of the field of view of this pulsar appears in Fig. 1.

Because of the presence of a $V=21.34$ star close to the position of PSR J1744-1134, the limiting magnitude for the pulsar counterpart was estimated by introducing artificial stars at the pulsar position in each filter, by using the IRAF task addstar, and repeating PSF-subtraction photometry (see Fig. 2). The limiting magnitudes found for 3.5- $\sigma$ detection by the automatic

\footnotetext{
${ }^{4}$ http://www.starlink.rl.ac.uk
}

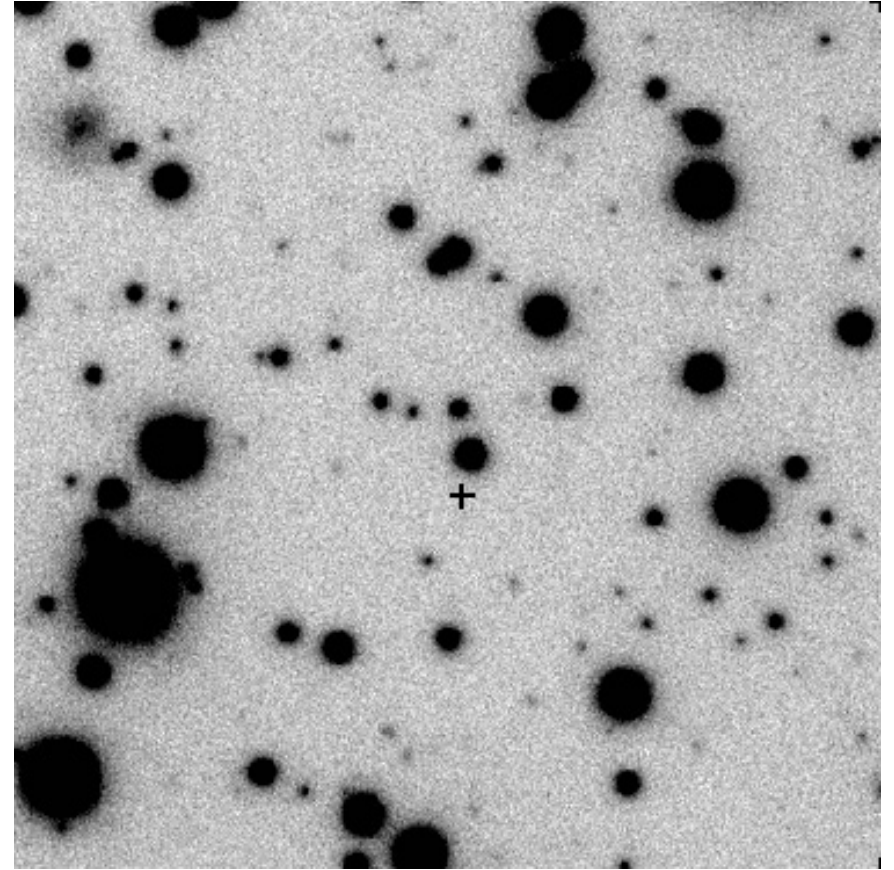

Fig. 1. VLT FORS1 V-band field of view $40^{\prime \prime} \times 40^{\prime \prime}$ centred about the radio timing position of PSR J1744-1139 (marked by the cross). North is to the top, and East to the left.

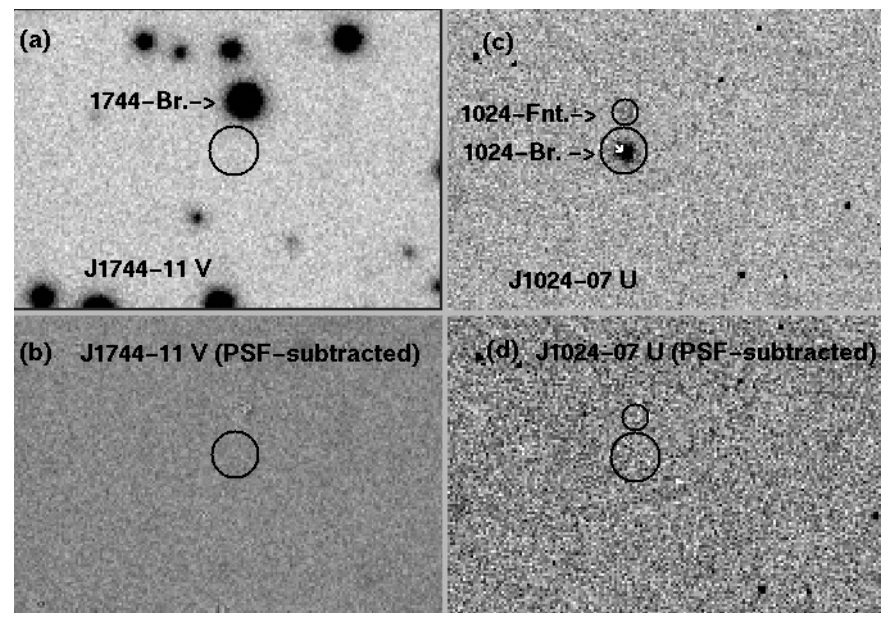

Fig. 2. Images a) and b) are the Field of View (FOV) of PSR J17441134 in $V$, before and after PSF-subtraction, respectively. The circle of $1^{\prime \prime}$ is centered at the radio position of the PSR to provide a scale. Images c) and d) show the FOV of PSR J1024-0719 in $U$, before and after PSF-subtraction respectively. The position of the pulsar within the 1 " circle is marked by the small white arrow. The blue object 1024-Fnt is encircled within the smaller region, just north of 1024-Br.

star-finding algorithm daophot are $B=26.9, V=26.3$, and $R=26.0$. We note that, from exposure-time considerations, assumed sky conditions, and a uniform input flux distribution (as a function of wavelength), the faintest object that could be detected in these frames would have $B=28.0, V=27.25$, $R=26.8$ for a $4-\sigma$ detection over the entire PSF, based on ESO FORS1 Exposure Time Calculator ${ }^{5}$ in its Imaging mode.

\footnotetext{
${ }^{5}$ http://www.eso.org/observing/etc
} 
For PSR J1024-0719, we find two objects within 1."5 of the radio position of the pulsar (see Fig. 2). The closer one, $1024-\mathrm{Br}$, only $\sim 0$.' 2 away, has magnitudes $U=22.11 \pm 0.03$, $V=19.82 \pm 0.01, R=18.89 \pm 0.01$, and $I=18.17 \pm 0.01$. The other, substantially fainter object, 1024-Fnt, $\sim 1$.' 5 north of the radio position, has magnitudes $U=23.8 \pm 0.1, V=24.9 \pm 0.1$, $R=24.4 \pm 0.1$ and $I=24.2 \pm 0.2$. The reported magnitude errors for both objects include the intrinsic error in the Landolt catalogue and the error in photometry. The angular separation between the bright and faint objects is 1.' 7 .

An object is reported close to the position of $1024-\mathrm{Br}$ in various optical and infrared catalogs. The USNO-A2 catalog (Monet et al. 1998) gives magnitudes $B=19.7$ and $R=19.1$, DENIS (DENIS Consortium, 1998) reports $I=18.0$, and 2MASS (IPAC/UMass, 2000) gives $J=17.2, H=16.1$, and $K=16.5$. Applying astrometric corrections based on coincident stars within our field, we find the DENIS and 2MASS positions to agree with our determination for 1024-Br to within 0.2 , whereas the USNO-A2 position is off by +1 .' $2^{\prime}$ in RA and +2 '. 0 in Dec. The similarity in positions and magnitudes makes it almost unavoidable that the source is the same in all cases. The discrepancy in the USNO-A2 position (taken in 1953, whereas all other measurements are from 1995-2001) may be either an error or an indication of a proper motion of -25 mas $\mathrm{yr}^{-1}$ in $\mathrm{RA}$ and -40 mas $\mathrm{yr}^{-1}$ in Dec, almost identical in direction, but about $40 \%$ lower in magnitude, compared to the radio-timing-determined proper motion of PSR J1024-0719.

Table 3. Positions and magnitudes of optical point sources closest to the radio positions of two MSPs. The limiting magnitudes are also provided for $3.5 \sigma$ detection at the central pixel in the exposure times used here for PSR J1744-1134.

\begin{tabular}{|c|c|c|c|}
\hline \multirow[t]{2}{*}{$\overline{\overline{\text { Object }}}$} & \multirow[t]{2}{*}{ Magnitude } & \multicolumn{2}{|c|}{ "PSR pos. - Obj. pos. } \\
\hline & & $\delta \mathrm{RA}$ & $\delta \mathrm{Dec}$ \\
\hline $\begin{array}{l}\text { J1744-1134 } \\
\text { (upper lim.) }\end{array}$ & $\begin{array}{c}B \geq 26.9, V \geq 26.3, \\
R \geq 26.0\end{array}$ & $0{ }^{\prime} 0$ & $0 .{ }^{\prime} 0$ \\
\hline $1744-\mathrm{Br}$ & $\begin{array}{c}B=22.86, V=21.34, \\
R=20.61\end{array}$ & $+0 ! 4$ & $-2{ }^{\prime \prime} 0$ \\
\hline $1024-\mathrm{Br}$ & $\begin{array}{c}U=22.11, V=19.82, \\
R=18.89, I=18.17\end{array}$ & $+0{ }^{\prime} 1$ & $-0 ! 2$ \\
\hline 1024-Fnt & $\begin{array}{c}U=23.8, V=24.9, \\
R=24.4, I=24.2\end{array}$ & -0.2 & $+1^{\prime \prime} 5$ \\
\hline
\end{tabular}

\subsection{Extinction-corrected magnitudes and multi-waveband fluxes}

Based on the dispersion measures of the two pulsars, BT99 obtained hydrogen column densities in the directions of PSR J1024-0719 and PSR J1744-1134 as $N_{\mathrm{H}}=2 \times 10^{20} \mathrm{~cm}^{-2}$ and $N_{\mathrm{H}}=1 \times 10^{20} \mathrm{~cm}^{-2}$ respectively. Using the scaling of de Boer et al. (1987) between visual extinction and hydrogen column density, $A_{V}=N_{\mathrm{H}} /\left(1.79 \times 10^{21}\right)$, we obtain $A_{V}=0.11$ for PSR J1024-0719. The wavelength-dependent continuum absorption in the main photometric bands (Allen 2000) then
Optical emission (near): Crab, PSR0540-69, Vela, PSR1024-0719

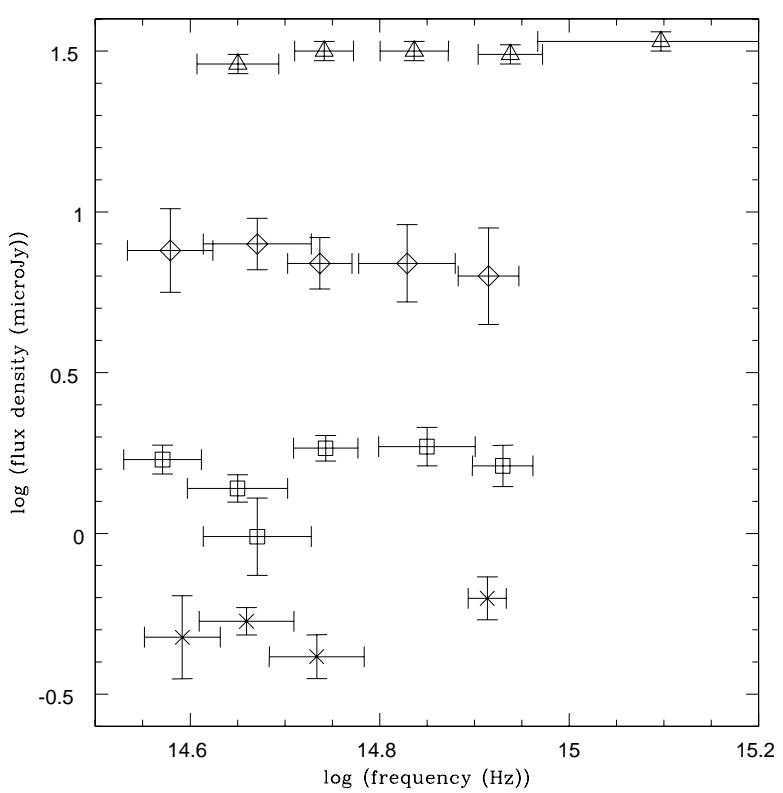

Fig. 3. Optical photometry of pulsars. The top set $(\Delta)$ is for the Crab pulsar with flux densities scaled down by a factor of 100 to fit in the graph. It also includes a measurement in the UV band at $\lambda=2400 \AA$. Next is the LMC pulsar PSR 0540-69 ( $\diamond)$, followed by the Vela pulsar PSR 0833-45 (squares). Finally, at the bottom are extinction-corrected flux densities of the object 1024-Fnt $(\times)$.

gives, for the same pulsar, $A_{U}=1.58 A_{V}=0.17, A_{R}=$ $0.749 A_{V}=0.08$, and $A_{I}=0.479 A_{V}=0.05$.

The extinction-corrected magnitudes for $1024-\mathrm{Br}$, assuming it to be at the same distance as the pulsar, are $U=21.94$, $V=19.71, R=18.81$, and $I=18.12$. Similarly, the extinctioncorrected magnitudes for 1024-Fnt are $U=23.65, V=24.83$, $R=24.33$ and $I=24.19$.

The corresponding extinction-corrected magnitude limits for PSR J1744-1134 are $B \geq 26.8$ (corresponding to $0.079 \mu \mathrm{Jy}$ at $B$-band centroid $\left.7.0 \times 10^{14} \mathrm{~Hz}\right), V \geq 26.2$, and $R \geq 26.0$.

\section{The optical objects close to PSR J1024-0719}

Since there are two point-like optical sources close to the nominal radio timing position of the pulsar, we examine their photometric colours and the spectrum of the brighter one to constrain their probable nature. The brighter star is in fact closer to the radio position of the pulsar, yet it is improbable that this is the optical counterpart.

\subsection{The brighter star $1024-\mathrm{Br}$}

The colours calculated for 1024-Br from the VLT observations, $V-R=0.93$ and $V-I=1.65$, do not match those of white dwarfs very well, but can be approximately accounted for by a K5-type main sequence dwarf (with $M \sim 0.7 M_{\odot}$, Allen 2000).

Because of the proximity of $1024-\mathrm{Br}$ to the radio position of the pulsar (corrected for proper motion), spectroscopic observations of this object were carried out. The spectra were acquired with the Boller \& Chivens spectrograph 


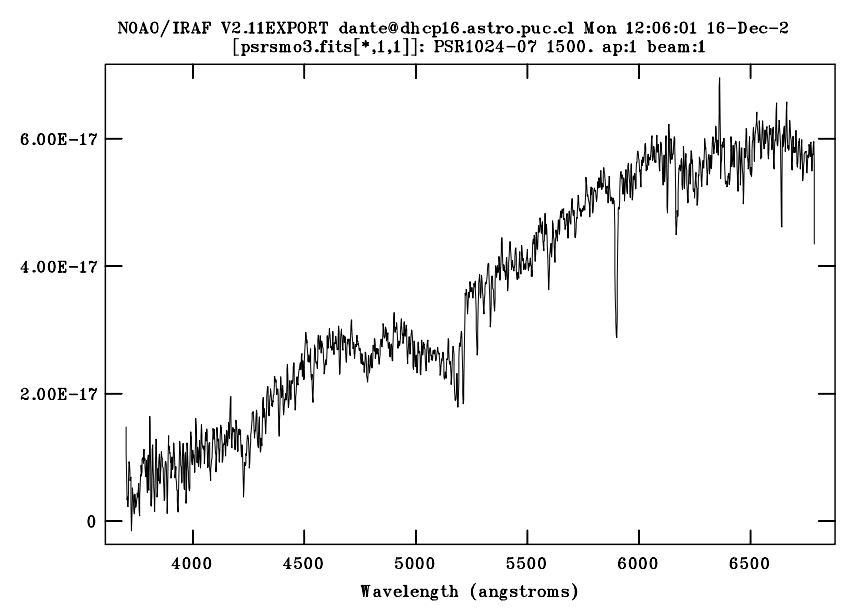

Fig. 4. Spectrum of the brighter star 1024-Br near PSR J1024-0719. The spectrum corresponds to a typical early K-type dwarf star.

on the Magellan I Baade $6.5 \mathrm{~m}$ telescope at Las Campanas Observatory on the night of May 8, 2002. These observations were taken during a spectroscopic survey of NGC 5128 globular clusters (see Minniti \& Rejkuba 2002 for more details on the reduction procedures). We obtained two exposures of the source 1024-Br with 900 and $1500 \mathrm{~s}$, respectively, at an airmass of 1.1. The measured seeing at the time of the observations was $0 . ' 8$, and a slit width of 1.'0 was used. The spectrophotometric standard LTT4816 observed at a similar airmass was used for the flux calibration, although the latter is uncertain due to the presence of thin cirrus during the night. The spectral reductions and measurements were carried out in IRAF, using the set of packages in CCDRED and TWODSPEC. The extracted spectra cover from 3700 to $6780 \AA$, with $1.5 \AA$ pix $^{-1}$. The final average spectrum corrected for cosmic rays has a mean $S / N=70 \mathrm{pix}^{-1}$, and is shown in Fig. 4. A few narrow absorption lines are present, and the spectrum corresponds to a typical early K-type dwarf star. Figure 4 shows absorption features due to the $\mathrm{Mg} \mathrm{H}$ band, the $\mathrm{Mg} 5170$ triplet, a weak $\mathrm{Ca} \mathrm{H}$ and $\mathrm{K}$ doublet, the $\mathrm{Na} \mathrm{D}$ doublet, as well as a number of iron lines such as Fe5270 and Fe5335. The lines of the hydrogen Balmer series are weak, and no prominent emission lines are present that would render this object peculiar in any way.

The tiny timing residuals $(<1 \mu \mathrm{s})$ from radio observations of PSR J1024-0719 (Toscano et al. 1999a) limit the mass of any binary companion to $m \sin i<10^{-3} M_{\oplus}$. Thus, the object $1024-\mathrm{Br}$ is unlikely to be a binary companion of the pulsar, and is most likely a near-solar-mass background star located far beyond the 200 pc upper limit for the pulsar distance.

\subsection{The faint object 1024-Fnt}

In the $M_{V}$ vs. $V-I$ plane, we compare 1024-Fnt with the cooling track for a $0.6 M_{\odot}$ white dwarf (DA or non-DA) given in Fig. 6 of Bergeron et al. (1995). The $V-I$ colour is 0.73 . Using $A_{V}=0.11$ as above, and the distance modulus $\mu=$ $5 \log (d / \mathrm{pc})-5+A_{V}=6.61$ for a distance of $200 \mathrm{pc}, 1024-$ Fnt would have an absolute magnitude $M_{V}=24.9-6.6=18.3$. This colour-magnitude position is well below the cooling curve of a $0.6 M_{\odot}$ white dwarf (both a DA or a non-DA, i.e., hydrogen-rich or hydrogen-less atmosphere white dwarf with a carbon core) as in Wood's (1990, 1995, as quoted in Bergeron et al. 1995) evolutionary model. The multi-band spectrum of this object and its possible nature are discussed in the next section.

\section{Implications for thermal and non-thermal models of the two neutron stars}

If we take a single blackbody spectral distribution over a whole neutron star surface, the deepest ( $B$-band) limit for PSR J1744-1134 (with $B \geq 26.9$ and $A_{B}=0.07$ ) corresponds to a temperature

$T_{\infty} \leq 2.1 \times 10^{6} \mathrm{~K}\left(\frac{d / 0.36 \mathrm{kpc}}{R_{\infty} / 10 \mathrm{~km}}\right)^{2}$,

where $T_{\infty}=T\left(1-2 G M / R c^{2}\right)^{1 / 2}$ and $R_{\infty}=R /\left(1-2 G M / R c^{2}\right)^{1 / 2}$ are the redshifted temperature and radius. The limits obtained here are consistent with those obtained for PSR J0108-1431 (Mignani et al. 2003), considering the respective exposures and distances. We note, however, that the X-ray detection of PSR J1744-1134 (BT99) already constrains this temperature to much lower values.

We plot the multi-band flux densities of the faint source 1024-Fnt near the radio pulsar PSR J1024-0719 as a function of the photon frequency in Fig. 3. The centroids and the bandpasses correspond to those of FORS1 Bessel filters (FORS1 2000). For comparison, we also plot in this figure the corresponding multi-band flux densities from the Crab (Percival et al. 1993), LMC pulsar PSR B0540-69 (Middleditch et al. 1987 as restated by Nasuti et al. 1997), and Vela (Mignani \& Caraveo 2001, dereddened fluxes from their Table 1). We note that 1024-Fnt has a spectral energy distribution similar to those of the well-known optical pulsars, as all objects have relatively flat spectra (with the exception of Vela near the $R$-band). For comparison, in other wave-bands, the spectral (energy) index of Crab, $\gamma\left(I_{v} \propto v^{-\gamma}\right)$ is consistent with $\gamma=0$ at optical frequencies and varies from $\gamma=1.1$ in the $\gamma$-ray region via 0.7 in the hard $\mathrm{X}$-rays to 0.5 in the soft $\mathrm{X}$-rays. The Crab spectral index changes sign and goes to $\gamma=-2$ in the far infrared. Its radio frequency component is a separate component from the higher energy part (flux density decreasing with increasing frequency again) and has $\gamma=2.7$ (Lyne \& Smith 1990).

Similarly, the X-ray flux density of PSR J1024-0719 in the ROSAT $0.1-2.4 \mathrm{keV}$ band and its standard error were computed from the absorption-corrected flux $f_{\mathrm{X}} \sim 2 \times$ $10^{-14} \mathrm{erg} \mathrm{s}^{-1} \mathrm{~cm}^{-2}$, reported by BT99, with an assumed photon index $\alpha=2.3(\gamma=1.3)$. We note that the X-ray emission from two MSPs as seen by Chandra (Zavlin et al. 2002) and by XMM-Newton (Becker \& Aschenbach 2002) must be described by composite models: for PSR J0030+0451 by a broken power-law ( $\left.\alpha_{1}=1.98-2.4\right)$ at energies below break $E_{\mathrm{b}}=1.28 \mathrm{keV}$, and for the binary PSR J0437-4715 by a power law $\alpha \sim 2$ together with a thermal spectrum of temperature $0.5-2 \times 10^{6} \mathrm{~K}$ from a heated polar cap. Thus, the power law assumed here is consistent with the observed power-law indices of MSPs. In the case of PSR J1024-0719, this gives a 
X-ray, optical and radio emission from near PSR1024-0719

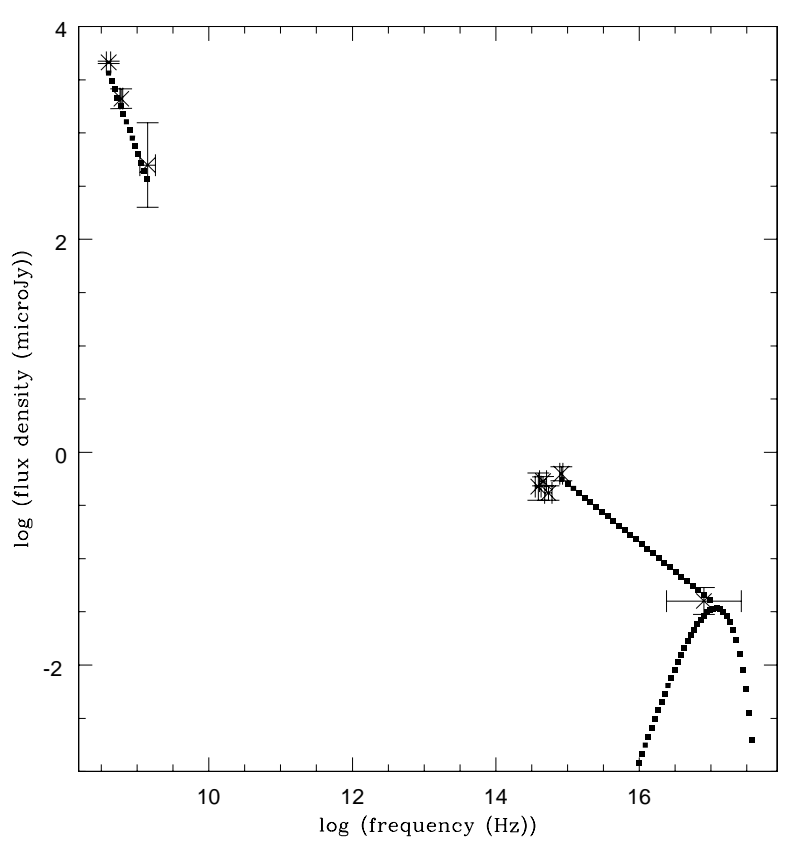

Fig. 5. Multiwaveband emission from near PSR J1024-0719. Included are the radio flux densities at 400,600 and $1400 \mathrm{MHz}$; the optical data (this paper) of 1024-Fnt; and the ROSAT HRI X-ray data. Two illustrative power laws and a blackbody curve are shown. The blackbody emission is from a heated region of the neutron star polar cap of radius $60 \mathrm{~m}$ and temperature $2 \times 10^{6} \mathrm{~K}$, at a distance of $50 \mathrm{pc}$.

flux density of $4.1 \times 10^{-2} \mu \mathrm{Jy}$ with the centroid at $0.33 \mathrm{keV}$ $\left(7.97 \times 10^{16} \mathrm{~Hz}\right)$ and an effective bandwidth of $0.23 \mathrm{keV}$. The radio flux densities at three frequencies $(400 \mathrm{MHz}, 600 \mathrm{MHz}$ and $1400 \mathrm{MHz}$ ) for both pulsars were obtained from Bailes et al. (1997). To guide the eye in Fig. 5 for the multi-band data, we also draw a few representative spectra: a) a power law with photon index $\alpha_{\mathrm{r}}=2.85(\gamma=1.85)$, which matches the radio data well; b) a power-law spectrum of index $\alpha_{\mathrm{oX}}=1.55$ connecting the highest frequency optical datum to the X-ray, and c) a blackbody spectrum of temperature $T_{\infty}=2.1 \times 10^{6} \mathrm{~K}$ from a heated cap $\left(R_{\infty}^{\text {core }}=0.06 \mathrm{~km}\right)$ of the neutron star surface at a distance of $d=0.2 \mathrm{kpc}$. It is clear that a single power law connecting the optical emission from 1024-Fnt and the X-ray emission from PSR J1024-0719 shall involve a minimum photon index of 1.55 and the maximum limit on the hot polar cap temperature $T \leq 2.1 \times 10^{6} \mathrm{~K}$ for a hot-spot of radius $60 \mathrm{~m}$. On the other hand, the optical magnitude in the $U$-band implies for a single blackbody function for the entire neutron star surface a temperature

$$
\begin{aligned}
T_{\infty} & \leq 4 \times 10^{4} \mathrm{~K} / \ln \left\{1+4.35 \times 10^{-4}\left[\left(R_{\infty} / 10 \mathrm{~km}\right) / d_{\mathrm{kpc}}\right]^{2}\right\} \\
& \approx 3.7 \times 10^{6} \mathrm{~K}\left(\frac{d / 0.2 \mathrm{kpc}}{R_{\infty} / 10 \mathrm{~km}}\right)^{2},
\end{aligned}
$$

where the latter expression corresponds to the Rayleigh-Jeans limit. This limit is again much less constraining than that obtained from the X-ray data of BT99.

\section{Discussion}

Since no MSP has yet been shown to be emitting optical pulses, only slower pulsars can act as guides for what may be expected from optical counterpart of a MSP. A comparison of a few key parameters between the two classes of pulsars is therefore of interest in this context. Non-thermal models for the optical radiation of slower pulsars like the Crab ascribe it to synchrotron emission (see Pacini 1971 and Pacini \& Salvati 1987) by relativistic particles near the light cylinder radius at very small pitch angles $(\Psi \ll 1 / \gamma$, where the Lorentz factor may be $\gamma \sim 10^{2}-10^{3}$; Crusius-Wätzel et al. 2001). Malov \& Machabeli (2001) show that finite pitch angles of the plasma particles appear in the outer magnetosphere near the light cylinder due to an instability associated with the cyclotron resonance condition being fulfilled there. They further show that pulsars with shorter periods such as the MSPs attain harder spectra and higher peak frequencies. Although MSPs have much smaller surface magnetic fields than slower pulsars, they also have smaller corotating magnetosphere radii, making the dipolar fields at light cylinder distances (a key parameter for particle acceleration models) similar to those of slower pulsars. Thus, in many of the MSPs, acceleration of particles in outer gaps sustained by pair creation by $\mathrm{GeV}$ gamma-rays may still be operative as in high spin-down luminosity pulsars (Cheng et al. 1986). The non-thermal power-law component of radiation due to pair-gap discharge is expected to scale with the Goldreich-Julian particle flux $\dot{N}_{\mathrm{GJ}}=(\boldsymbol{\Omega} \cdot \boldsymbol{B}) / 2 \pi e$ from the magnetosphere (see Harding 1981 or Thompson 1998). For some MSPs, $\dot{N}_{\mathrm{GJ}}$ is in excess of that of PSR B0656+14, which is found to be a gamma-ray, X-ray, and optical pulsar. We note that for our target pulsars, the Goldreich-Julian particle fluxes are close to that of Geminga (within a factor of two), from which optical pulsations have possibly been seen (Shearer et al. 1998).

Scaled to the period ( $P=5.16 \mathrm{~ms}$ ) and the surface magnetic field $\left(B_{0} \sim 10^{8.1} \mathrm{G}\right)$ in PSR J1024-0719, expressions (33) and (35) of Crusius-Wätzel et al. (2001) imply:

$$
\frac{I_{v}^{\mathrm{opt}}}{I_{v}^{\mathrm{X}}}=6 \times 10^{-2} \frac{\left(\Psi_{\mathrm{opt}} / 10^{-3}\right)^{2}\left(v_{\mathrm{opt}} / 10^{15} \mathrm{~Hz}\right)(P / 5.16 \mathrm{~ms})^{3 / 2}}{\left(\Psi_{\mathrm{X}} / 0.1\right)^{3 / 2}\left(v_{\mathrm{X}} / 10^{17} \mathrm{~Hz}\right)^{1 / 2}\left(B_{0} / 10^{8.1} \mathrm{G}\right)^{1 / 2}}
$$

If we assume that the object 1024-Fnt is indeed the pulsar optical counterpart, and the observed emission is primarily pulsed, then $I^{\text {opt }} / I^{\mathrm{X}} \sim 15$ instead of $6 \times 10^{-2}$ for PSR J1024-0719 (see Fig. 5). Thus, it is possible that in MSPs the average pitch angles responsible for optical radiation are larger, $\Psi_{\mathrm{opt}} \sim 10^{-2}$.

\section{Conclusions}

So far, none of the isolated MSPs has been shown to pulse in the optical. Deep exposures carried out with the VLT of the field of the nearby, isolated pulsar PSR J1744-1134 in multiple bands do not show a plausible optical counterpart. We derive upper limits to the optical emission of this pulsar and the surface temperature of the underlying neutron star at its measured distance. In the field of view of PSR J1024-0719, we detect two objects close to the radio position of the pulsar. We argue, 
based on photometry and spectroscopy, that the brighter object is a background early K-type dwarf star. The object 1024-Fnt has a multi-band spectrum which is unusual and characteristic of pulsars, such as the Crab, Vela, and PSR B0540-69. Given its proximity to the radio position of PSR J1024-0719 and the considerable astrometric uncertainty, only timing analysis with high time resolution can unambiguously define its nature.

Acknowledgements. We thank Maria Cristina Depassier for her role in the formation of this collaboration and Marina Rejkuba for sharing with us the Magellan I spectroscopic data obtained in collaboration with D. Minniti. A. Ray thanks Malvin Ruderman for discussions on optical radiation from pulsars at the Aspen Center for Physics and Poonam Chandra for her comments on the manuscript. A. Reisenegger thanks Marten van Kerkwijk for advice about astrometry with the VLT, Frédéric Courbin for help with the "p2pp" and René Méndez for useful discussions. We thank George Pavlov as well as an anonymous referee for their comments on the manuscript which helped us to improve this paper. We acknowledge the use of the Guide Star Catalogue II, which is a joint project of the Space Telescope Science Institute and the Osservatorio Astronomico di Torino. We have also used the software packages provided by the Starlink Project, which is based at Rutherford Appleton Laboratory and funded by PPARC. The USNO-A2.0 catalog is distributed by the U.S. Naval Observatory Flagstaff Station under the Precision Measuring machine project. F. Sutaria is presently an Alexander von Humboldt Fellow at the Technische Universität München, Garching. A. Reisenegger's and G. Hertling's work was supported by FONDECYT grant No. 1020840, and H. Quintana's and D. Minniti's by the FONDAP Center for Astrophysics, grant No. 15010003. At Tata Institute, this work was a part of the Five Year Plan Projects 10P-201 and 9P-208[a].

\section{References}

Allen, C. W. 2000, Allen's Astrophysical Quantities (4th. edition), ed. A. N. Cox (Springer)

Alpar, M. A., Cheng, A., Ruderman, M. A., \& Shaham, J. 1982, Nature, 300, 728

Bailes, M., Johnston, S., Bell, J. F., et al. 1997, ApJ, 481, 386

Becker, W., \& Aschenbach, B. 2002, in Proceedings of the 270 WE-Heraeus Seminar on Neutron Stars, Pulsars and Supernova Remnants, ed. W. Becker, H. Lesch, \& J. Trümper, MPE Report, 278, 64 [astro-ph/0208466]

Becker, W., \& Trümper, J. 1997, A\&A, 326, 682

Becker, W., \& Trümper, J. 1999, A\&A, 341, 803-817: BT99

Bergeron, P., Wesemael, F., \& Beauchamp, A. 1995, PASP, 107, 1047

de Boer, K. S., Jura, M. A., \& Shull, J. M. 1987, in Exploring the Universe with IUE, ed. Y. Kondo (D. Reidel \& Company)

Cheng, K. S., Chau, W. Y., Zhang, J. L., \& Chau, H. F. 1992, ApJ, 396, 135

Cheng, K. S., Ho, C., \& Ruderman, M. A. 1986, ApJ, 300, 500

Cocke, W. J., Disney, M. J., \& Taylor, D. J. 1969, Nature, 221, 525

Crusius-Wätzel, A. R., Kunzl, T., \& Lesch, H. 2001, ApJ, 546, 401

Deutsch, E. W. 1999, AJ, 118, 1882

Fomalont, E. B., Goss, W. M., Manchester, R. N., Lyne, A. G., \& Justtanont, K. 1992, MNRAS, 258, 497
Fomalont, E. B., Goss, W. M., Manchester, R. N., \& Lyne, A. G. 1997, MNRAS, 286, 81

FORS1+2 User Manual, Paranal Observatory Very Large Telescope, European Southern Observatory Manual, 87

Harding, A. 1981, ApJ, 245, 267

Hewish, A., Bell, S. J., Pilkington, J. D. H., Scott, P. F., \& Collins, R. A. 1968, Nature, 217, 709

Koptsevich, A. B., Lundqvist, P., Serafimovich, N. I., Shibanov, Yu. A., \& Sollerman, J. 2003, A\&A, 400, 265

Landolt, A. U. 1992, AJ, 104, 340

Larson, M. B., \& Link, B. 1999, ApJ, 521, 271

Lyne, A. G., \& Graham-Smith, F. 1990, Pulsar astronomy (Cambridge Univ. Press)

Ma, C., Arias, E. F., Eubanks, T. M., et al. 1998, AJ, 116, 516

Malov, I., \& Machabeli, G. Z. 2001, ApJ, 554, 587

Massey, P., \& Davis, L. E. 1992, A Users guide to stellar photometry IRAF

Middleditch, J., Pennypacker, C. R., \& Burns, M. S. 1987, ApJ, 315, 142

Mignani, R. P., \& Becker, W. 2003 [astro-ph/0301114]

Mignani, R. P., \& Caraveo, P. A. 2001, A\&A, 376, 213

Mignani, R. P., Manchester, R. N., \& Pavlov, G. G. 2003, ApJ, 582, 978

Minniti, D., \& Rejkuba, M. 2002, ApJ, 575, L59

Monet, D., Bird, A., Canzian, B., et al. 1998, Vizier On-line Data Catalog: I/252, U.S. Naval Observatory Flagstaff Station (USNOFS)

Nasuti, F. P., Mignani, R., Caraveo, P. A., \& Bignami, G. F. 1997, A\&A, 323, 839

Pacini, F. 1971, ApJ, 163, L17

Pacini, F., \& Salvati, M. 1987, ApJ, 321, 447

Pavlov, G. G., Stringfellow, G. S., \& Cordova, F. A. 1996, ApJ, 467, 370

Percival, J. W., Biggs, J. D., Dolan, J. F., et al. 1993, ApJ, 407, 276

Reisenegger, A. 1995, ApJ, 442, 749

Rots, A. H., Jahoda, K., Macomb, D., et al. 1998, ApJ, 501, 749

Ruderman, M. A., \& Sutherland, P. G. 1975, ApJ, 196, 51

Shklovskii, I. S. 1970, Soviet Astron., 13, 562

Shearer, A., \& Golden, A. 2001, ApJ, 547, 967

Shearer, A., \& Golden, A. 2002, in Neutron stars, Pulsars and Supernova Remnants, WE-Heraeus Seminar, ed. W. Becker et al., MPE Report, 278, 44

Shearer, A., Golden, A., Harfst, S., et al. 1998, A\&A, 335, L21

Staelin, D. H., \& Reifenstein, E. C. 1968, Science, 162, 1481

Taylor, J. H., \& Cordes, J. M. 1993, ApJ, 411, 674

Thompson, D. J. 1998, in Neutron stars and pulsars, ed. N. Shibazaki et al. (Tokyo: Universal Academic Press)

Toscano, M., Sandhu, J. S., Bailes, M., et al. 1999a, MNRAS, 307, 925

Toscano, M., Britton, M. C., Manchester, R. N., et al. 1999b, ApJ, 523, L171

Wood, M. A. 1990, Ph.D. Thesis, University of Texas at Austin

Wood, M. A. 1995, in White Dwarfs, Lecture Notes in Physics, ed. D. Koester, \& K. Werner (Berlin, Heidelberg, New York: Springer-Verlag), 443, 41

Zavlin, V. E., Pavlov, G. G., Sanwal, D., et al. 2002, ApJ, 569, 894 